\title{
FKBP12 is a predictive biomarker for efficacy of anthracycline-based chemotherapy in breast cancer
}

\author{
Mingyou Xing ${ }^{2} \cdot$ Jun Wang ${ }^{1} \cdot$ Qin Yang ${ }^{1} \cdot$ Yu Wang $^{1} \cdot$ Jiansha $\mathrm{Li}^{1} \cdot$ Jing Xiong ${ }^{1} \cdot$ Sheng Zhou ${ }^{1}$
}

Received: 11 May 2019 / Accepted: 6 August 2019 / Published online: 19 August 2019

(C) The Author(s) 2019

\begin{abstract}
Background FK506-binding protein 12 (FKBP12) is abundant, ubiquitously expressed cytoplasmic protein with multiple functions in cell signaling transduction. Recently, we reported a novel function for FKBP12 in oncoprotein mouse double minute 2 (MDM2) self-ubiquitination and degradation, which greatly enhanced the sensitivity of cancer cells to chemotherapy. However, the clinical relevance remains unclear.

Methods An immunohistochemical analysis of FKBP12 expression was performed in a cohort of 524 patients with invasive breast cancer. The correlations of FKBP12 expression with patient survival and chemoresponse were statistically analyzed. MDA-MB-468 cells were transfected with FKBP12 siRNA or Myc-tagged FKBP12, and then, the tumor cells were treated with doxorubicin followed by western blot, cell viability, and apoptosis assay.

Results The expression of FKBP12 was decreased in breast cancer tissues, and there was a significant correlation between FKBP12 loss and MDM2 overexpression. Furthermore, FKBP12 loss was specifically correlated with poor prognosis and increased resistance to anthracycline-based chemotherapy. Kaplan-Meier survival analysis showed that overall survival (OS) and disease-free survival (DFS) were both significantly lower in the low FKBP12 expression group than those in the high FKBP12 expression group. In patients treated with anthracycline-based preoperative chemotherapy, low FKBP12 expression patients had a significant lower rate of pathologic complete response (pCR). Importantly, these results seemed to be driven mainly by MDM2. These observations were especially prominent in the MDM2-positive subgroup. Univariate and multivariate analyses revealed that FKBP12 loss was an independent factor for predicting prognosis and pCR. In in vitro assay, FKBP12 silence led to significant upregulation of MDM2. Accordingly, MDA-MB-468 cells with FKBP12 silence were less responsive to doxorubicin-induced cytotoxic and apoptotic effect. In contrast, in FKBP12-transfected MDA-MB-468 cells, MDM2 was more greatly inhibited by doxorubicin, resulting in greater cytotoxic and apoptotic effect.

Conclusions We propose that FKBP12 loss, which can be enhanced by MDM2 overexpression, predicts poor prognosis and chemoresistance. Increasing the expression of FKBP12 may be a valuable strategy to add to anthracycline-based chemotherapy, especially in MDM2-overexpressed patients.
\end{abstract}

Keywords Breast cancer $\cdot$ FKBP12 $\cdot$ MDM2 $\cdot$ Prognosis $\cdot$ Chemoresistance

Jing Xiong

xiongjingtj@126.com

$\triangle$ Sheng Zhou zhou71@163.com

Mingyou Xing

xingmingyou@126.com

Jun Wang

106402445@qq.com

Qin Yang

70918404@qq.com
Yu Wang

tongjisiyu@163.com

Jiansha Li

lijiansha2018@163.com

1 Institute of Pathology, Tongji Hospital, Tongji Medical College, Huazhong University of Science and Technology, Wuhan 430030, China

2 Department of Infectious Disease, Tongji Hospital, Tongji Medical College, Huazhong University of Science and Technology, Wuhan 430030, China 


$\begin{array}{ll}\text { Abbreviations } \\ \text { FKBP12 } & \text { FK506-binding protein } 12 \\ \text { MDM2 } & \text { Mouse double minute } 2 \\ \text { OS } & \text { Overall survival } \\ \text { DFS } & \text { Disease-free survival } \\ \text { TNBC } & \text { Triple-negative breast cancer } \\ \text { pCR } & \text { Pathologic complete response } \\ \text { mTOR } & \text { Mammalian target of rapamycin } \\ \text { RyR } & \text { Ryanodine receptor } \\ \text { TGF- } \beta \text { RI } & \text { Transforming growth factor- } \beta \text { type I receptor } \\ \text { EGFR } & \text { Epidermal growth factor receptor } \\ \text { AJCC } & \text { American Joint Committee on Cancer } \\ \text { NPI } & \text { Nottingham prognostic index }\end{array}$

\section{Background}

Chemotherapy remains the mainstay for human breast cancer treatment. Especially for triple-negative breast cancer (TNBC), with no drug-targetable receptors, cytotoxic chemotherapy is the only recommended therapeutic regimen [1-3]. However, only certain patients respond well to this cytotoxic chemotherapy. Therefore, the treatment of breast cancer, especially TNBC, is a significant challenge. Reliable biomarkers that predict tumor response to chemotherapy are quite valuable when making treatment decisions, and new therapeutic strategies against the corresponding molecular pathways associated with chemoresistance are urgently required to improve clinical outcome.

FK506-binding protein 12 (FKBP12) is a $12-\mathrm{kDa}$ protein that is abundantly and ubiquitously expressed mainly in cytosol and possesses peptidyl prolyl cis-trans isomerase activity [4]. It can bind the immunosuppressants FK506 and rapamycin. When bound to FK506, FKBP12 forms a ternary complex with calcineurin to inhibit the serine/threonine phosphatase activity of calcineurin and interfere with the signal transduction in activated $T$ lymphocytes $[5,6]$. In complex with rapamycin, FKBP12 interacts with mammalian target of rapamycin (mTOR) and inhibits its roles in regulating cell growth and cancer progression $[7,8]$. In the absence of FK506, FKBP12 binds to other different cellular receptors or targets such as ryanodine receptor (RyR), which is one of the major calcium release channels in the sarcoplasmic and endoplasmic reticula. Interaction between FKBP12 and RyR stabilizes RyR channel and modulates channel gating by increasing channel full conductance levels and mean open time [9]. FKBP12 has also been shown to interact with transforming growth factor- $\beta$ type I receptor (TGF- $\beta$ RI) to inhibit receptor-mediated signal transduction [10]. In addition, FKBP12 has an inhibitory effect on the cellular activity of epidermal growth factor receptor (EGFR) by modulating the receptor's phosphorylation status [11].
Recently, we reported a novel function for FKBP12 in oncoprotein mouse double minute 2 (MDM2) self-ubiquitination and degradation, which greatly enhanced the sensitivity of cancer cells to chemotherapy [12]. Specifically, FKBP12 binds to the C-terminal RING domain of MDM2 protein to disrupt heterodimer formation with MDM4 and induces its E3 ligase activity for self-ubiquitination. When cancer cells are subjected to doxorubicin treatment, the increased expression of MDM2 following activation of p53 is also markedly inhibited by FKBP12. This is because p53 induces MDM2 translocation from the nucleus to the cytoplasm, where facilitates FKBP12/MDM2 interaction. FKBP12-mediated degradation of MDM2 confers continuing and constitutive activation of $\mathrm{p} 53$, suppression of XIAP, and consequent sensitization of cancer cells to the cytotoxic and apoptotic effects of doxorubicin. However, the clinical relevance of FKBP12 remains unclear. As chemotherapy is still the mainstay of treatment for human breast cancer, we herein examined the expression of FKBP12 by immunohistochemistry in breast cancer. We revealed that there was indeed a significant correlation between FKBP12 and MDM2 expression and that the expression level of FKBP12 in cancer tissue might predict prognosis and response to chemotherapy.

\section{Materials and methods}

\section{Patients}

This study was approved by the Ethics Committee of Tongji Hospital, Huazhong University of Science and Technology. A cohort of 524 patients with invasive breast cancer was included in this study. All patients were diagnosed and treated at Tongji Hospital between September 2007 and January 2012. The patients ranged from 32 to 78 years of age (median, 56 years). Detailed clinicopathologic characteristics of these patients are summarized in Table 1. Tumor was graded according to a modified Bloom-Richardson scoring system. Clinicopathologic staging was based on the American Joint Committee on Cancer (AJCC) Cancer Staging Manual. The Nottingham prognostic index $(N P I)$, which is calculated using the size of the lesion $(S)$, the number of involved lymph nodes $(N)$, and the grade of the tumor $(G)$, was used to determine patient prognosis $(N P I=(0.2 \times S)+N+G)$. According to gene expression, patients were divided into the following three distinct molecular subtypes: Luminal (ER+ and HER2-); HER2+; and TNBC (ER-, PR-, and HER2-). Follow-up data were collected from clinical records, ranging from 5 to 72 months post-diagnosis (median, 48 months). Overall survival (OS) was the period from diagnosis to the date of breast cancerrelated death, while disease-free survival (DFS) was the 
Table 1 Clinicopathologic variables in relation to FKBP12 and MDM2 immunoreactivity in invasive breast cancer and TNBC

\begin{tabular}{|c|c|c|c|c|c|c|c|}
\hline Variable & Total $(n=524)$ & $\begin{array}{l}\text { FKBP12 high, no. } \\
(\%)\end{array}$ & $\begin{array}{l}\text { FKBP12 low, no. } \\
(\%)\end{array}$ & $P$ value & $\begin{array}{l}\text { MDM2 high, no. } \\
(\%)\end{array}$ & $\begin{array}{l}\text { MDM2 low, sno. } \\
(\%)\end{array}$ & $P$ value \\
\hline \multicolumn{8}{|l|}{ Age } \\
\hline$<56$ & 313 & $85(27.2)$ & $228(72.8)$ & \multirow[t]{2}{*}{0.748} & $189(60.4)$ & $124(39.6)$ & \multirow[t]{2}{*}{0.471} \\
\hline$\geq 56$ & 211 & $60(28.4)$ & 151 (71.6) & & $134(63.5)$ & $77(36.5)$ & \\
\hline \multicolumn{8}{|l|}{ Menopausal status } \\
\hline Premenopausal & 265 & $81(30.6)$ & $184(69.4)$ & \multirow[t]{2}{*}{0.134} & $156(58.9)$ & $109(41.1)$ & \multirow[t]{2}{*}{0.187} \\
\hline Postmenopausal & 259 & $64(24.7)$ & $195(75.3)$ & & $167(64.5)$ & $92(35.5)$ & \\
\hline \multicolumn{8}{|l|}{ Size-TNM } \\
\hline pT1 & 188 & $59(31.4)$ & 129 (68.6) & \multirow[t]{3}{*}{0.109} & $106(56.4)$ & $82(43.6)$ & \multirow[t]{3}{*}{0.112} \\
\hline $\mathrm{pT} 2+\mathrm{pTx}$ & 252 & $70(27.8)$ & $182(72.2)$ & & $159(63.1)$ & $93(36.9)$ & \\
\hline $\mathrm{pT} 3+\mathrm{pT} 4$ & 84 & $16(19.0)$ & $68(81.0)$ & & $58(69.0)$ & $26(31.0)$ & \\
\hline \multicolumn{8}{|l|}{ Histologic grade } \\
\hline Grade I & 97 & $33(34.0)$ & $64(66.0)$ & \multirow[t]{3}{*}{0.264} & $48(49.5)$ & $49(50.5)$ & \multirow[t]{3}{*}{0.015} \\
\hline Grade II & 217 & $61(28.1)$ & $156(71.9)$ & & $135(62.2)$ & $82(37.8)$ & \\
\hline Grade III & 210 & $51(24.3)$ & 159 (75.7) & & $140(66.7)$ & $70(33.3)$ & \\
\hline \multicolumn{8}{|l|}{ LN metastasis } \\
\hline Positive & 319 & $83(26.0)$ & $236(74.0)$ & \multirow[t]{2}{*}{0.291} & 204 (63.9) & $115(36.1)$ & \multirow[t]{2}{*}{0.175} \\
\hline Negative & 205 & $62(30.2)$ & $143(69.8)$ & & $119(58.0)$ & $86(42.0)$ & \\
\hline \multicolumn{8}{|l|}{$N P I$} \\
\hline$<3.4$ & 180 & $57(31.7)$ & $123(68.3)$ & \multirow[t]{3}{*}{0.107} & $84(46.7)$ & $96(53.3)$ & \multirow[t]{3}{*}{$<0.001$} \\
\hline $3.4-5.4$ & 269 & $74(27.5)$ & $195(72.5)$ & & $189(70.3)$ & $80(29.7)$ & \\
\hline$>5.4$ & 75 & $14(18.7)$ & $61(81.3)$ & & $50(66.7)$ & $25(33.3)$ & \\
\hline \multicolumn{8}{|l|}{ MIB-1 (\%) } \\
\hline$<10$ & 204 & $63(30.9)$ & $141(69.1)$ & \multirow[t]{3}{*}{0.134} & $117(57.4)$ & 87 (42.6) & \multirow[t]{3}{*}{0.028} \\
\hline $10-30$ & 254 & $70(27.6)$ & $184(72.4)$ & & $171(67.3)$ & $83(32.7)$ & \\
\hline$>30$ & 66 & $12(18.2)$ & $54(81.8)$ & & $35(53.0)$ & $31(47.0)$ & \\
\hline $\mathrm{ER} \alpha$ & & & & & & & \\
\hline Positive & 343 & $107(31.2)$ & $236(68.8)$ & 0.013 & $200(58.3)$ & $143(41.7)$ & 0.031 \\
\hline Negative & 181 & $38(21.0)$ & $143(79.0)$ & & $123(68.0)$ & $58(32.0)$ & \\
\hline PR & & & & & & & \\
\hline Positive & 319 & $98(30.7)$ & $221(69.3)$ & 0.052 & 187 (58.6) & $132(41.4)$ & 0.076 \\
\hline Negative & 205 & 47 (22.9) & $158(77.1)$ & & $136(66.3)$ & $69(33.7)$ & \\
\hline HER2 amplificatio & & & & & & & \\
\hline Positive & 103 & $21(20.4)$ & $82(79.6)$ & 0.065 & $71(68.9)$ & $32(31.1)$ & 0.090 \\
\hline Negative & 421 & $124(29.5)$ & $297(70.5)$ & & $252(59.9)$ & $169(40.1)$ & \\
\hline Hormone therapy & & & & & & & \\
\hline No & 182 & $41(22.5)$ & $141(77.5)$ & 0.120 & $121(66.5)$ & $61(33.5)$ & 0.114 \\
\hline Yes (Tamoxifen) & 332 & $102(30.7)$ & $230(69.3)$ & & $198(59.6)$ & $134(40.4)$ & \\
\hline Unknown & 10 & $2(20.0)$ & $8(80.0)$ & & $4(40.0)$ & $6(60.0)$ & \\
\hline Chemotherapy & & & & & & & \\
\hline No & 31 & $9(29.0)$ & $22(71.0)$ & 0.235 & $19(61.3)$ & $12(38.7)$ & 0.118 \\
\hline Yes (FEC) & 469 & $133(28.4)$ & $336(71.6)$ & & 294 (62.7) & $175(37.3)$ & \\
\hline Unknown & 24 & $3(12.5)$ & $21(87.5)$ & & $10(41.7)$ & $14(58.3)$ & \\
\hline Recurrence & & & & & & & \\
\hline No & 387 & $115(29.7)$ & $272(70.3)$ & 0.079 & $228(58.9)$ & $159(41.1)$ & 0.031 \\
\hline Yes & 137 & $30(21.9)$ & $107(78.1)$ & & $95(69.3)$ & $42(30.7)$ & \\
\hline MDM2 expression & & & & & & & \\
\hline High & 323 & $81(25.1)$ & $242(74.9)$ & 0.092 & & & \\
\hline Low & 201 & $64(31.8)$ & $137(68.2)$ & & & & \\
\hline
\end{tabular}


Table 1 (continued)

\begin{tabular}{|c|c|c|c|c|c|c|c|}
\hline Variable & Total $(n=524)$ & $\begin{array}{l}\text { FKBP12 high, no. } \\
(\%)\end{array}$ & $\begin{array}{l}\text { FKBP12 low, no. } \\
(\%)\end{array}$ & $P$ value & $\begin{array}{l}\text { MDM2 high, no. } \\
(\%)\end{array}$ & $\begin{array}{l}\text { MDM2 low, sno. } \\
(\%)\end{array}$ & $P$ value \\
\hline \multicolumn{8}{|c|}{ FKBP12 expression } \\
\hline High & 145 & & & & $81(55.9)$ & $64(44.1)$ & 0.092 \\
\hline Low & 379 & & & & $242(63.9)$ & $137(36.1)$ & \\
\hline \multicolumn{8}{|c|}{ Molecular subtype } \\
\hline $\begin{array}{l}\text { Luminal (non } \\
\text { HER2+) }\end{array}$ & 334 & $102(30.5)$ & $232(69.5)$ & 0.144 & $196(58.7)$ & $138(41.3)$ & 0.171 \\
\hline HER2+ & 102 & $24(23.5)$ & $78(76.5)$ & & $67(65.7)$ & $35(34.3)$ & \\
\hline TNBC & 88 & 19 (21.6) & $69(78.4)$ & & $60(68.2)$ & $28(31.8)$ & \\
\hline Variable & $\begin{array}{l}\text { TNBC } \\
(n=88)\end{array}$ & $\begin{array}{l}\text { FKBP12 high, no. } \\
(\%)\end{array}$ & $\begin{array}{l}\text { FKBP12 low, no. } \\
(\%)\end{array}$ & $P$ value & $\begin{array}{l}\text { MDM2 high, no. } \\
(\%)\end{array}$ & $\begin{array}{l}\text { MDM2 low, sno. } \\
(\%)\end{array}$ & $P$ value \\
\hline MDM2 high & 60 & 7 (11.7) & $53(88.3)$ & 0.001 & & & \\
\hline MDM2 low & 28 & $12(42.9)$ & $16(57.1)$ & & & & \\
\hline FKBP12 high & 19 & & & & $7(36.8)$ & $12(63.2)$ & 0.001 \\
\hline FKBP12 low & 69 & & & & $53(76.8)$ & $16(23.2)$ & \\
\hline
\end{tabular}

$L N$ lymph node, NPI Nottingham Prognostic Index, ER $\alpha$ estrogen receptor, PR progesterone receptor, HER2 human epidermal growth factor receptor 2, FEC 5-fluorouracil + epirubicin + cyclophosphamide, $T N B C$ triple-negative breast cancer

period from diagnosis to the date of breast cancer-derived relapse or metastasis.

Of the 524 invasive breast cancer patients, 166 (including $67 \mathrm{TNBC}$ ) patients received six cycles of anthracycline-based neoadjuvant chemotherapy (FEC 100 regimen: 5-florouracil $500 \mathrm{mg} / \mathrm{m}^{2}$, epirubicin $100 \mathrm{mg} / \mathrm{m}^{2}$, cyclophosphamide $500 \mathrm{mg} /$ $\mathrm{m}^{2}$, once every 21 days). A core needle biopsy was performed prior to chemotherapy for pathologic diagnosis and other biologic evaluation. Patients underwent mastectomy and axillary lymph-node excision or breast-conserving surgery to assess tumor response about 4 weeks after the sixth cycle of chemotherapy. Pathologic complete remission (pCR) was defined as disappearance of invasive tumor lesion in the surgically removed breast and axillary lymph nodes after chemotherapy.

\section{Immunohistochemistry}

The formalin-fixed paraffin-embedded archival tissue block was cut into $4-\mu \mathrm{m}$-thick tissue sections. Only tissue samples collected prior to chemotherapy were eligible. The sections were deparaffinized in xylene and subsequently rehydrated through a graded series of ethanol. Endogenous peroxidase was blocked in 3\% hydrogen peroxide for $15 \mathrm{~min}$. Antigen retrieval was performed in $0.01 \mathrm{M}$ citrate buffer $(\mathrm{pH} 6.0)$ at $100{ }^{\circ} \mathrm{C}$ for $20 \mathrm{~min}$. Immunohistochemistry was performed by Envision method. The sections were first incubated with the primary antibody anti-FKBP12 (N-19; 1:100; Santa Cruz Biotechnology, Santa Cruz, CA, USA) or anti-MDM2 (SMP14; 1:200; Sigma-Aldrich, St. Louis, MO, USA) at $4{ }^{\circ} \mathrm{C}$ overnight. Negative and blank control samples were run accordingly. After being rinsed three times in PBS, secondary antibody (Envision, Anti-Goat/Mouse-HRP, DAKO, Glostrup, Denmark) was applied to all sections and incubated at $37{ }^{\circ} \mathrm{C}$ for $1 \mathrm{~h}$, followed by three PBS rinses. Finally, the sections were developed with diaminobenzidine (DAB) and counterstained with hematoxylin.

Staining results were evaluated by three independent pathologists blinded to the patient details. FKBP12 shows cytoplasmic staining, while MDM2 shows nuclear staining with or without cytoplasmic staining. FKBP12 and MDM2 expression were scored using a modified histochemical score (H-score). $\mathrm{H}$-score includes both the intensity of staining and the percentage of stained cells. The intensity of staining was scored $0-3(0=$ no staining, $1=$ weak staining, $2=$ moderate staining, and $3=$ strong staining). The percentage of stained cells was estimated (0-100\%). H-score (range from 0 to 300) was calculated by multiplication of the two indices. Using a cut-off score of 100, FKBP12 or MDM2 expression was categorized into two groups: low expression $(\mathrm{H}$-score $<100)$ and high expression (H-score $\geq 100)$.

\section{Cell culture, plasmid transfection, and doxorubicin treatment}

MDA-MB-468 cells were obtained from the China Center for Type Culture Collection (CCTCC). siFKBP12 and Myctagged FKBP12 plasmids were kindly provided by Prof. Muxiang Zhou (Emory University School of Medicine, Atlanta, GA, USA). Doxorubicin was purchased from Sigma-Aldrich. MDA-MB-468 cells were transfected with FKBP12 siRNA or Myc-tagged FKBP12 using Lipofectamine 2000 transfection kit from Invitrogen (CarIsbad, CA, USA), followed by 
treatment with different concentrations of doxorubicin. The cells were collected for western blot, cell viability, and apoptosis assay.

\section{Western blot}

Western blot, performed by standard procedures, was used to measure the protein expression levels of FKBP12 and MDM2 in tumor cells. The following antibodies were used: anti-FKBP12 (N-19; 1:1000; Santa Cruz Biotechnology), antiMyc (9B11; 1:1000; Cell Signaling Technology, Danvers, MA, USA), and anti-MDM2 (SMP14; 1:1000; Sigma-Aldrich). GAPDH served as an internal control.

\section{WST-1 assay}

Cell viability was detected by WST-1 assay. Cells with FKBP12 siRNA or Myc-tagged FKBP12 transfection were grown in 96-well plates, treated with different concentrations of doxorubicin for $24 \mathrm{~h}$, and incubated with WST-1 reagent (Roche, Mannheim, Germany) for an additional $4 \mathrm{~h}$. The optical density was read using a microplate reader.

\section{Flow cytometry}

Cell apoptosis was detected by flow cytometry. Cells with FKBP12 siRNA or Myc-tagged FKBP12 transfection and doxorubicin treatment were stained with FITC-Annexin V and propidium iodide (BD Pharmingen, San Diego, CA, USA). Stained cells were detected on a FACScan flow cytometer.

\section{Statistical analysis}

All data were analyzed using SPSS statistical software. The Chi-square and Fisher's exact tests were used to examine the correlations between FKBP12 or MDM2 expression and different clinicopathologic variables. The Spearman rank correlation coefficient test was used to evaluate the correlations between FKBP12 and MDM2 in breast cancer tissue. Survival curves were plotted using the Kaplan-Meier method, and the significance of observed differences was determined using the log-rank test. The influence of each variable on survival was tested with the Cox proportional hazard regression model. $P<0.05$ was considered statistically significant.

\section{Results}

\section{FKBP12 and MDM2 protein expression pattern in breast cancer tissue}

FKBP12 protein expression was observed in the cytoplasm of epithelial cells of normal breast ducts and lobules.
However, FKBP12 protein expression was decreased in breast cancer tissues. Of the 524 invasive breast cancers, 145 (27.7\%) showed high immunoreactivity (moderate-to-strong staining), while 238 (45.4\%) showed low immunoreactivity (weak staining) and 141 (26.9\%) showed an absence of staining. MDM2 protein showed absent or weak expression in normal breast tissues. However, immunopositive reaction for MDM2 was detected in $323(61.6 \%)$ of the 524 invasive breast cancers. Subcellular localization of MDM2 protein in cancer cells was predominantly nuclear with or without cytoplasmic. Representative images of FKBP12 and MDM2 expression are presented in Fig. 1.

\section{FKBP12 and MDM2 expression correlated with clinicopathologic parameters}

We correlated FKBP12 and MDM2 expression to patient clinicopathologic characteristics such as age at diagnosis, tumor size, histologic grade, lymph-node metastatic status, NPI, proliferation rate of breast cancer cells (Index of MIB-1), ER, PR, HER2, and molecular subtype. The correlations are summarized in Table 1. Loss of FKBP12 was significantly correlated with ER negativity $(P=0.013)$. MDM2 overexpression was also significantly correlated with ER negativity $(P=0.031)$. There was no significant correlation found between FKBP12 or MDM2 expression and HER 2 amplification $(P>0.05)$. According to gene expression, patients were divided into three different molecular subtypes: luminal (ER+ and HER2-); HER2+; and TNBC (ER-, PR-, and HER2-). Our results showed that FKBP12 or MDM2 expression was not differently represented in any of these particular molecular subtypes $(P>0.05)$. In addition, MDM2 overexpression was found to be significantly correlated with higher histologic grade $(P=0.015)$, higher NPI $(P<0.001)$, higher index of MIB-1 $(P=0.028)$, and recurrence $(P=0.031)$. No significant correlation occurred between FKBP12 expression and these parameters $(P>0.05)$.

\section{FKBP12 loss correlated with MDM2 overexpression}

Using Spearman rank correlation coefficient test, we studied the correlation of FKBP12 with MDM2. There was a trend toward correlation of FKBP12 loss with MDM2 overexpression, but the difference did not reach statistical significance in the overall population $(n=524, P>0.05)$ (Table 1). However, in TNBC patients, we found a significant correlation between FKBP12 loss and MDM2 overexpression $(n=88, P=0.001)$ (Table 1$)$. 

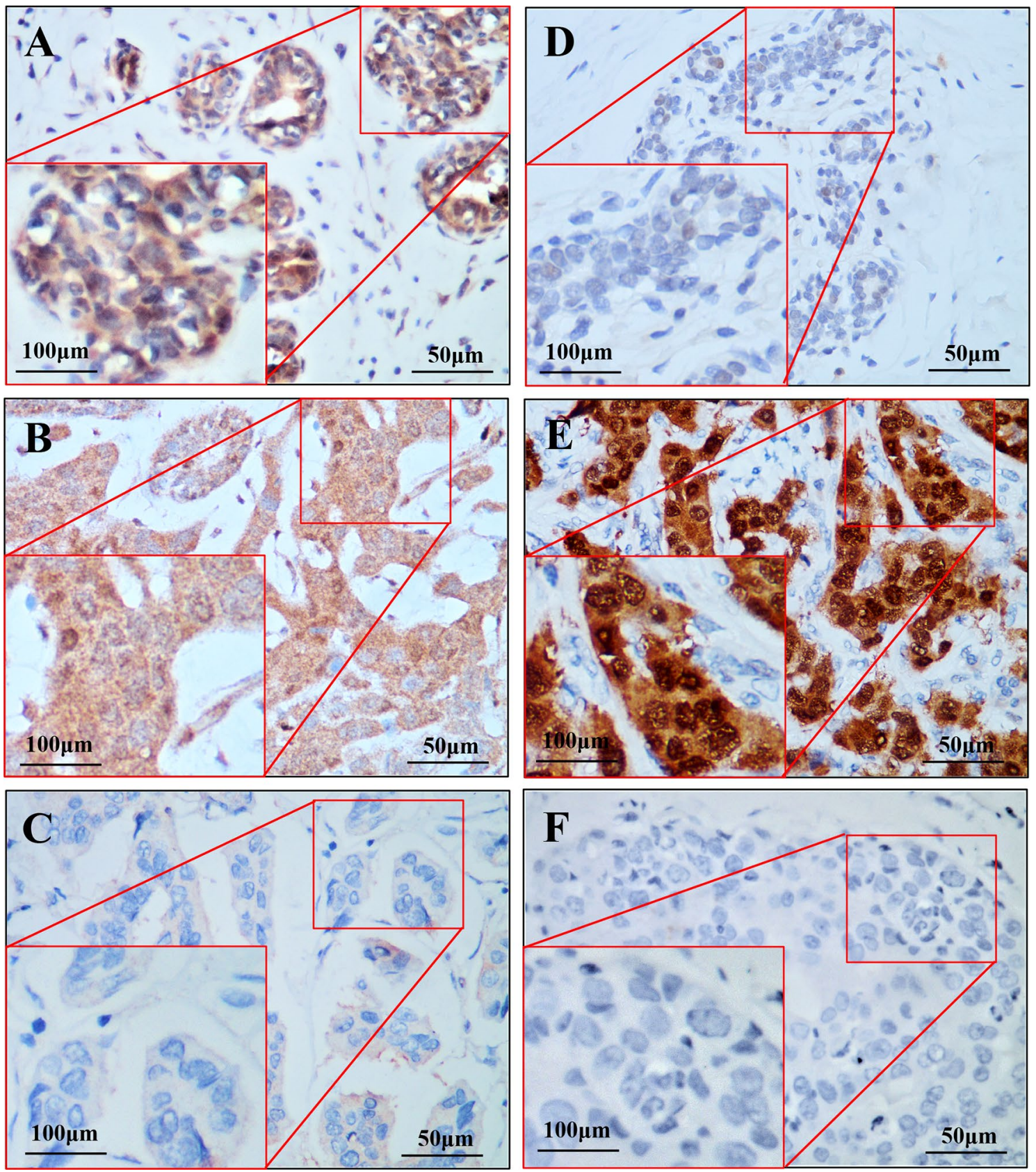

Fig. 1 Representative images of immunohistochemical staining for FKBP12 and MDM2 protein. a FKBP12-positive staining in benign breast tissue. b FKBP12 high staining in invasive breast cancer. c FKBP12 extremely low staining in invasive breast cancer. d MDM2

\section{Correlation of FKBP12 expression with clinical outcome}

Next, we investigated the correlation of FKBP12 expression with clinical outcome to study the clinical significance of FKBP12. Kaplan-Meier survival analysis was performed in the overall population and different molecular subtypes of low staining in benign breast tissue. e MDM2 high staining in invasive breast cancer. $f$ MDM2-negative staining in invasive breast cancer

breast cancer. As shown in Fig. 2, FKBP12 loss indicated a worse clinical outcome. OS and DFS were both significantly lower in the low FKBP12 expression group than those in the high FKBP12 expression group. FKBP12 loss was significantly correlated with worse OS and DFS in TNBC patients ( $n=88, P=0.0009$ and $P=0.0007$, respectively) (Fig. 2d), but not in the overall population $(n=524, P>0.05)$ (Fig. 2a), 
Fig. 2 Overall survival and disease-free survival curves according to FKBP12 expression in all patients (a), luminal patients (b), HER2+ patients (c), and TNBC patients (d)
A

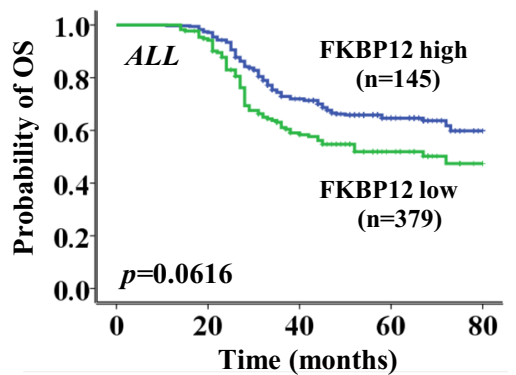

B

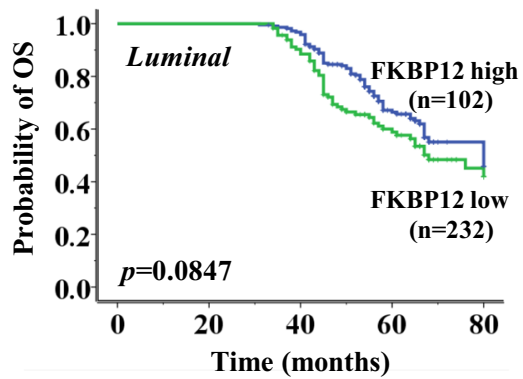

C

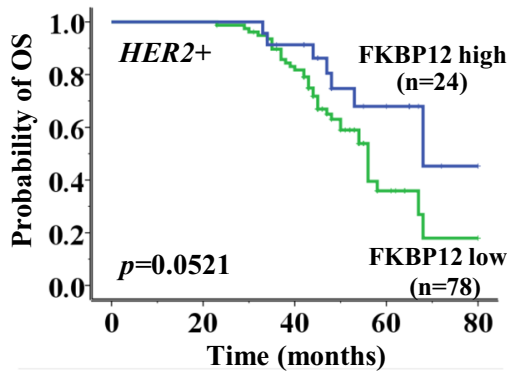

D

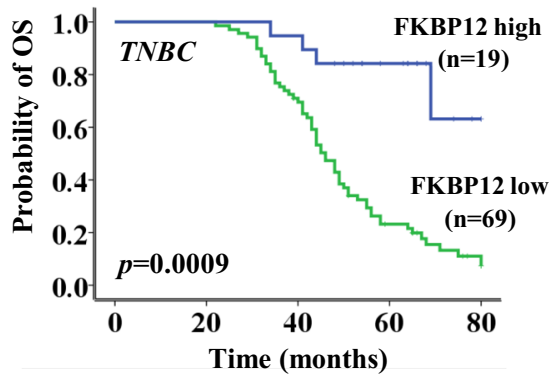

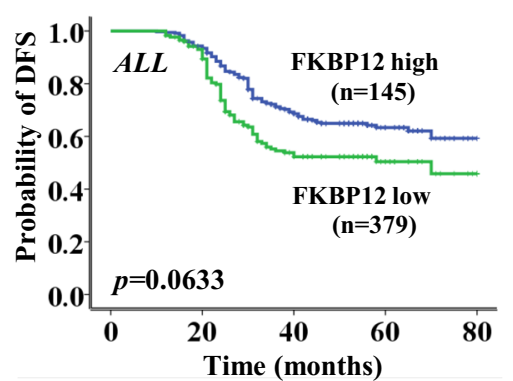
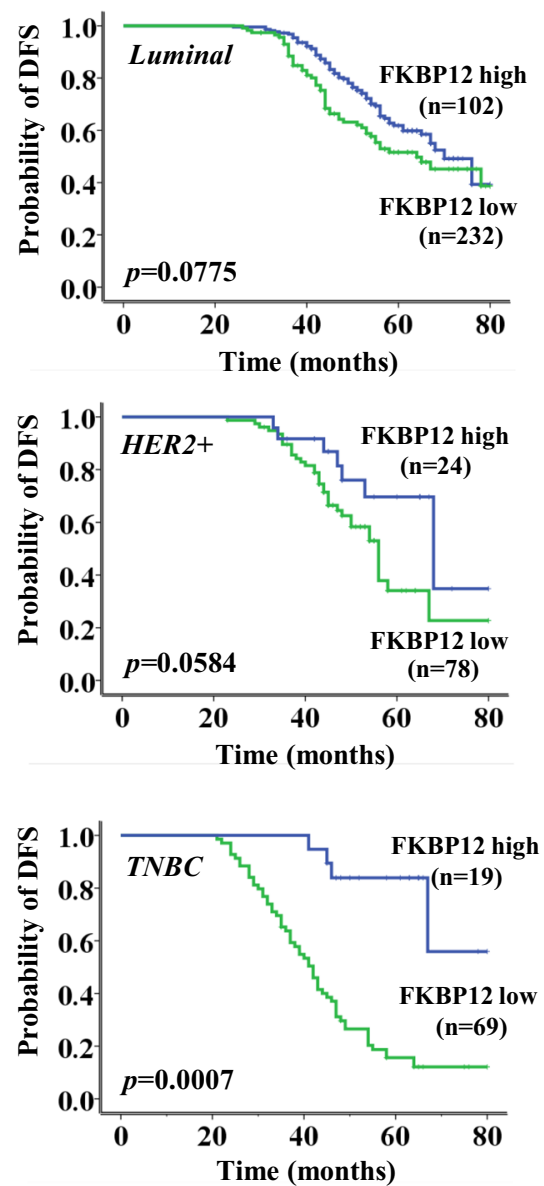

in patients with luminal breast cancer $(n=334, P>0.05)$ (Fig. 2b), or in patients with HER2+ breast cancer $(n=102$, $P>0.05$ ) (Fig. 2c). Interestingly, the result seemed to be driven mainly by MDM2. This observation was especially prominent in the MDM2-positive subgroup (OS, $P=0.0004$ for MDM2-positive subgroup and $P=0.0013$ for MDM2negative subgroup; DFS, $P<0.0001$ for MDM2-positive subgroup and $P=0.0011$ for MDM2-negative subgroup) (Fig. 3a, b). Cox multivariate analysis revealed that, independent of lymph-node involvement and MDM2 overexpression, FKBP12 loss was an independent prognostic factor for TNBC patients in OS and DFS $(P<0.001$ and $P<0.001$, respectively) (Table 2).

\section{Response to chemotherapy according to FKBP12 expression}

Anthracyclines are among the most commonly used chemotherapeutic agents for breast cancer. However, only some patients respond well to anthracycline-based chemotherapy. Of the 524 invasive breast cancer patients, 166 received anthracycline-based neoadjuvant chemotherapy. We investigated further the correlation of FKBP12 expression with the efficacy of anthracycline-based neoadjuvant chemotherapy. We analyzed FKBP12 expression in biopsies taken at diagnosis and its relationship with pathologic complete remission (pCR), defined as the disappearance of 
Fig. 3 Overall survival and disease-free survival curves according to FKBP12 expression in MDM2-negative TNBC patients (a) and MDM2-positive TNBC patients $(\mathbf{b})$
A

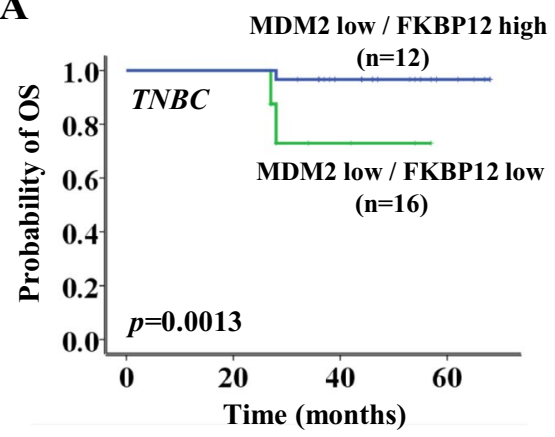

B

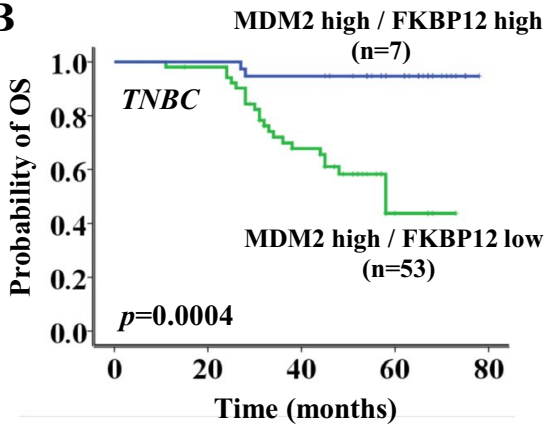

MDM2 low / FKBP12 high
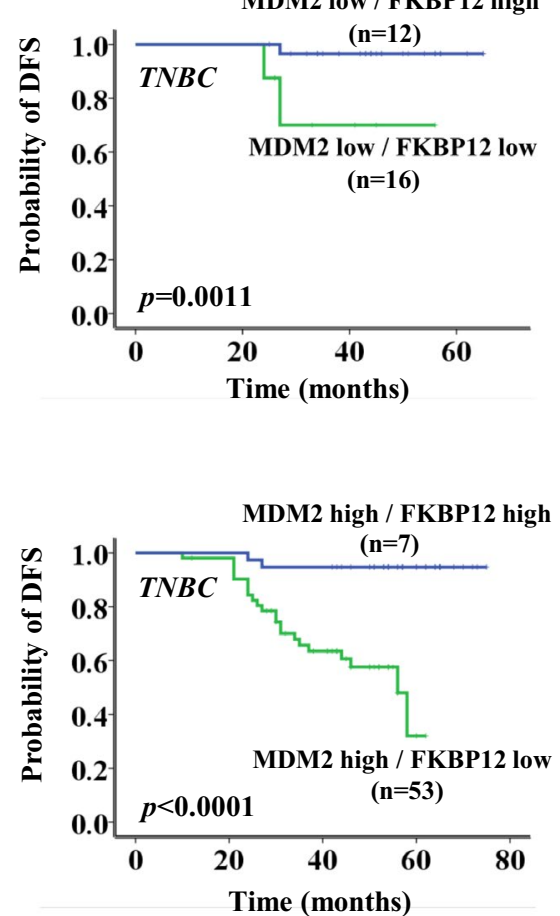

Table 2 Multivariate Cox model analysis of disease-free survival and overall survival in TNBC

\begin{tabular}{|c|c|c|c|c|c|c|}
\hline \multirow[t]{2}{*}{ Variable } & \multicolumn{3}{|c|}{ Disease-free survival } & \multicolumn{3}{|c|}{ Overall survival } \\
\hline & Hazard ratio & $95 \%$ CI & $P$ value & Hazard ratio & $95 \% \mathrm{CI}$ & $P$ value \\
\hline Age $(<56$ vs $\geq 56)$ & 0.834 & $0.433-1.748$ & 0.776 & 0.903 & $0.389-2.124$ & 0.786 \\
\hline Menopausal status (pre. vs post.) & 0.687 & $0.325-1.975$ & 0.477 & 0.525 & $0.223-1.867$ & 0.335 \\
\hline Size (pT1-2 vs pT3-4) & 0.659 & $0.236-1.682$ & 0.065 & 0.387 & $0.233-1.407$ & 0.059 \\
\hline Histological grade (I-II vs III) & 0.412 & $0.237-1.531$ & 0.158 & 0.704 & $0.498-1.994$ & 0.086 \\
\hline LN metastasis (Posi. vs Neg.) & 0.627 & $0.247-1.033$ & 0.014 & 0.754 & $0.162-1.228$ & 0.019 \\
\hline$N P I(<3.4$ vs $\geq 3.4)$ & 1.632 & $1.114-3.152$ & 0.113 & 0.603 & $0.327-1.178$ & 0.076 \\
\hline MIB $(<10 \%$ vs $\geq 10 \%)$ & 0.631 & $0.443-0.987$ & 0.069 & 0.836 & $0.530-1.625$ & 0.097 \\
\hline FKBP12 (high vs low) & 0.512 & $0.201-0.739$ & $<0.001$ & 0.317 & $0.186-0.586$ & $<0.001$ \\
\hline MDM2 (high vs low) & 0.405 & $0.427-0.855$ & 0.003 & 0.372 & $0.264-0.679$ & 0.005 \\
\hline
\end{tabular}

invasive tumor lesion in surgically removed breast and axillary lymph nodes after chemotherapy. As shown in Table 3, tumor response to chemotherapy was significantly correlated with the expression status of FKBP12. Low FKBP12 expression patients had a significant lower rate of $\mathrm{pCR}$. The pCR rate was significantly lower in the low FKBP12 expression group than that in the high FKBP12 expression group ( $n=166, P=0.006)$. Similarly, among the subtypes, FKBP12 loss correlated with lower pCR in TNBC patients $(n=67, P=0.003)$, but not in patients with luminal $(n=65$, $P>0.05)$ or HER $2+$ breast cancer $(n=34, P>0.05)$. This observation was also especially prominent in the MDM2positive subgroup $(P<0.001$ for MDM2-positive subgroup
Table 3 Correlation between chemoresponse and FKBP12 expression by intrinsic cancer subtype

\begin{tabular}{lrlr}
\hline Subtypes & $n$ & $\begin{array}{l}\text { PCR rate } \\
\text { (FKBP12 high/ } \\
\text { low) }\end{array}$ & $P$ value \\
\hline All patients & 166 & $47.0 \% / 15.1 \%$ & 0.006 \\
Luminal (non HER2+) & 65 & $33.8 \% / 18.5 \%$ & 0.412 \\
HER2+ & 34 & $38.2 \% / 17.6 \%$ & 0.294 \\
TNBC & 67 & $64.2 \% / 10.4 \%$ & 0.003 \\
TNBC =67 & & & \\
MDM2 high expression & 45 & $71.1 \% / 11.1 \%$ & $<0.001$ \\
MDM2 low expression & 22 & $50.0 \% / 9.10 \%$ & 0.005 \\
\hline
\end{tabular}


and $P=0.005$ for MDM2-negative subgroup) (Table 3). These data further supported the above prognostic results (Figs. 2,3), where anthracycline was also included in the adjuvant chemotherapy of most of the patients. In addition, in univariate and multivariate analyses, FKBP12 loss was an independent predictor against $\mathrm{pCR}$ for the overall population $(P=0.002$ and $P=0.004$, respectively) (Table 4$)$ and TNBC patients ( $P=0.003$ and $P=0.009$, respectively) (Table 5). Therefore, FKBP12 loss in breast cancer patients, especially in TNBC patients, was specifically associated with increased resistance to anthracycline-based chemotherapy.

\section{FKBP12 sensitizes breast cancer cells to chemotherapy}

We also investigated whether FKBP12 expression confers sensitivity to chemotherapy in breast cancer cells. We performed FKBP12 silence or transfection along with doxorubicin treatment in MDA-MB-468 cells and tested the MDM2 expression and cell survival. Western blot assay showed that the cellular expression of MDM2 was negatively regulated by FKBP12. FKBP12 silence led to significant upregulation of MDM2. Doxorubicin inhibited MDM2 expression in control MDA-MB-468 cells, but not in MDA-MB-468 cells with FKBP12 silence (Fig. 4a). Accordingly, MDA-MB-468 cells with FKBP12 silence were less responsive to doxorubicin-induced cytotoxic and apoptotic effect than control MDA-MB-468 cells (Fig. 4b, c). In contrast, in FKBP12transfected MDA-MB-468 cells, MDM2 was more greatly inhibited by doxorubicin (Fig. 4d), resulting in greater cytotoxic and apoptotic effect (Fig. 4e, f).

\section{Discussion}

MDM2 is a multifunctional oncoprotein and is overexpressed in various human cancers, including human breast cancer [13]. Overexpression of MDM2 in tumors correlates
Table 5 Univariate and multivariate analyses of pCR against various characteristics in TNBC patients

\begin{tabular}{llll}
\hline Variable & pCR rate (\%) & $\begin{array}{l}\text { Univariate } \\
\text { analysis } P \\
\text { value }\end{array}$ & $\begin{array}{l}\text { Multivariate } \\
\text { analysis } P \\
\text { value }\end{array}$ \\
\hline FKBP12 (high/low) & $64.2 / 10.4$ & 0.003 & 0.009 \\
MDM2 (high/low) & $19.4 / 35.8$ & 0.042 & 0.105 \\
Size (pT1-2/pT3-4) & $41.5 / 43.2$ & 0.642 & 0.466 \\
$\begin{array}{l}\text { Histological grade } \\
\quad 3-\text { II/III) }\end{array}$ & $39.5 / 44.7$ & 0.407 & 0.637 \\
LN metastasis (posi/ & $45.3 / 39.1$ & 0.373 & 0.578 \\
$\quad$ neg) & & & \\
NPI $(<3.4 / \geq 3.4)$ & $38.3 / 46.4$ & 0.175 & 0.233 \\
MIB $(<10 \% / \geq 10 \%)$ & $36.4 / 52.8$ & 0.043 & 0.153 \\
\hline
\end{tabular}

with disease progression and predicts a poor treatment outcome [14]. It has been well established that the oncogenic function of MDM2 protein is mainly through inhibiting the tumor suppressor p53 (restraining p53-mediated transcription and promoting p53 ubiquitination). The MDM2 gene is itself a transcript target of p53, and MDM2 and p53 form a mutual negative-feedback loop. When cells are in normal growing or in unstressed status, the MDM2-p53 negative-feedback loop is intact. However, in response to cellular stress and DNA damage such as treatment with certain chemotherapeutic drugs, p53 is rapidly accumulated and activated, inducing not only p21 and PUMA for cellcycle arrest and apoptosis but also increased transcription of MDM2. This p53-induced increase in MDM2 expression inhibits p53, which is an important mechanism for the development of chemoresistance [15-18]. MDM2 also plays p53-independent roles. In the absence of functional p53, tumor cells that express high levels of MDM2 still show high invasive potential [19]. P53-independent activities and mechanisms of MDM2 have attracted increasing attention in recent years. A recent series of studies, including ours, indicate that MDM2 is able to interact with other cellular
Table 4 Univariate and multivariate analyses of $\mathrm{pCR}$ against various characteristics in all patients

\begin{tabular}{llll}
\hline Variable & pCR rate $(\%)$ & $\begin{array}{l}\text { Univariate analysis } \\
P \text { value }\end{array}$ & $\begin{array}{l}\text { Multivariate analysis } \\
P \text { value }\end{array}$ \\
\hline FKBP12 (high/low) & $47.0 / 15.1$ & 0.002 & 0.004 \\
MDM2 (high/low) & $21.4 / 33.8$ & 0.038 & 0.065 \\
Size (pT1-2/pT3-4) & $27.2 / 29.4$ & 0.484 & 0.507 \\
Histological grade (I-II/III) & $24.6 / 30.8$ & 0.371 & 0.438 \\
LN metastasis (posi/neg) & $28.2 / 26.2$ & 0.673 & 0.328 \\
NPI (<3.4/ $\geq 3.4)$ & $21.4 / 32.4$ & 0.039 & 0.107 \\
MIB (<10\%/ $\geq 10 \%)$ & $22.8 / 31.5$ & 0.071 & 0.337 \\
ER $\alpha$ (posi/neg) & $22.5 / 36.1$ & 0.023 & 0.045 \\
PR (posi/neg) & $19.7 / 35.4$ & 0.028 & 0.047 \\
HER2 amplification (posi/neg) & $29.7 / 26.3$ & 0.336 & 0.503 \\
Molecular subtype (TNBC/others) & $41.8 / 17.2$ & 0.003 & 0.035 \\
\hline
\end{tabular}


A

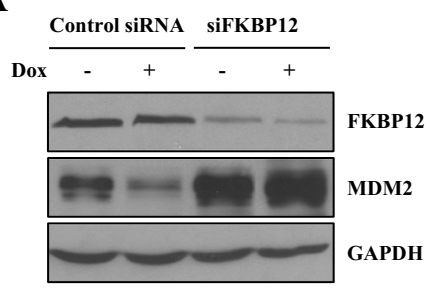

D

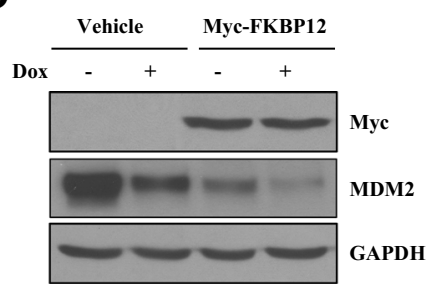

B

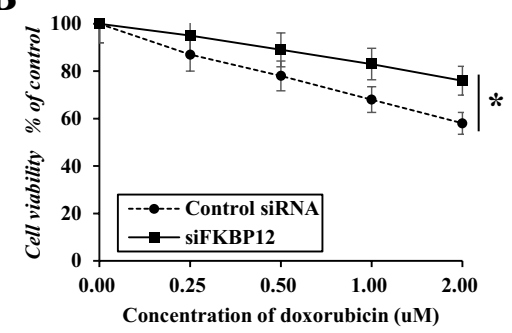

$\mathbf{E}$

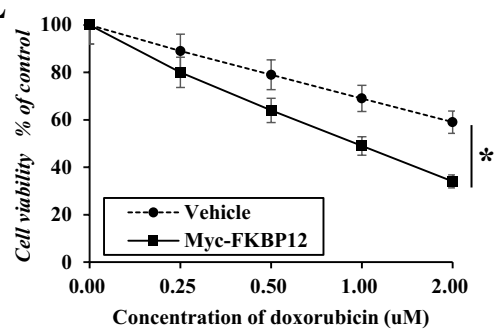

C

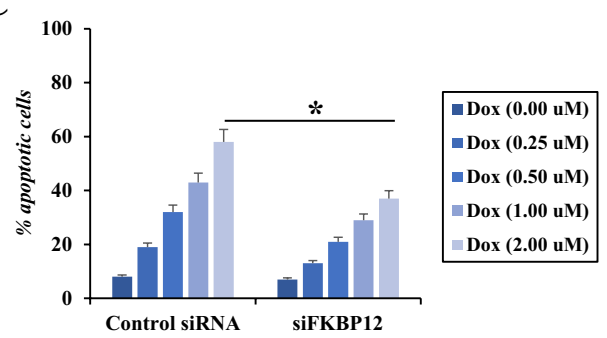

$\mathbf{F}$
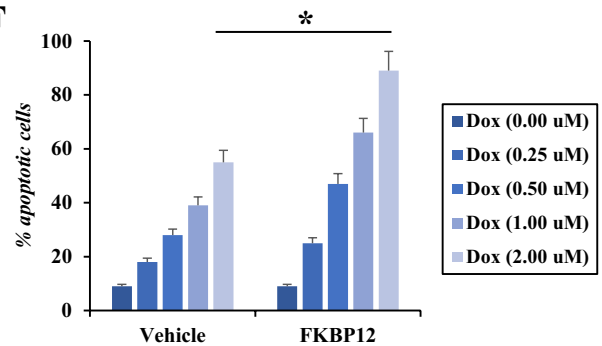

Fig. 4 Effect of FKBP12 on cell response to doxorubicin. MDAMB-468 cells were transfected with FKBP12 siRNA or Myc-tagged FKBP12, and then, the tumor cells were treated with different con-

molecules involved in tumor promotion and metastasis, such as XIAP [20, 21], VEGF [22, 23], and Akt [24, 25].

Recently, we reported that FKBP12 interacted with MDM2 and induced MDM2 ubiquitination and degradation [12]. FKBP12-mediated degradation of MDM2 confers continuing and constitutive activation of $\mathrm{p} 53$, suppression of XIAP, and consequent sensitization of cancer cells to the cytotoxic and apoptotic effects of doxorubicin. P53 is a classic tumor suppressor, while XIAP is an important member of the inhibitor-of-apoptosis protein (IAP) family. Therefore, FKBP12-mediated degradation of MDM2 should be clinically significant. Cytotoxic chemotherapy, particularly anthracycline-based chemotherapy regimens, is currently the mainstay of treatment for human breast cancer. We herein examined the expression of FKBP12 by immunohistochemistry in breast cancer and analyzed its correlation with anthracycline efficacy. Results suggest that the expression level of FKBP12 in breast cancer tissue can serve as a predictive biomarker of therapeutic outcome. Low FKBP12 expression was specifically correlated with poor prognosis and increased resistance to anthracycline-based chemotherapy. Kaplan-Meier survival analysis showed that OS and DFS were both significantly lower in the low FKBP12 expression group than those in the high FKBP12 expression group. In patients treated with anthracyclinebased preoperative chemotherapy, low FKBP12 expression patients had a significant lower rate of pCR. Importantly, these results seemed to be driven mainly by MDM2. These observations were especially prominent in the MDM2-positive subgroup. Finally, we performed FKBP12 silence or centrations of doxorubicin for $24 \mathrm{~h}$ followed by western blot (a, d), cell viability $(\mathbf{b}, \mathbf{e})$, and apoptosis assay $(\mathbf{c}, \mathbf{f})$. Data represent mean \pm SEM of three independent experiments. ${ }^{*} P<0.05$

transfection in MDA-MB-468 breast cancer cells to confirm whether FKBP12 regulates MDM2 expression and confers sensitivity to chemotherapy. FKBP12 silence led to significant upregulation of MDM2. Accordingly, MDA-MB-468 cells with FKBP12 silence were less responsive to doxorubicin-induced cytotoxic and apoptotic effect than control MDA-MB-468 cells. In contrast, in FKBP12-transfected MDA-MB-468 cells, MDM2 was more greatly inhibited by doxorubicin, resulting in greater cytotoxic and apoptotic effect.

As mentioned, the MDM2-p53 feedback loop strongly operates leading to chemoresistance. In contrast, FKBP12mediated degradation of MDM2 inhibits the MDM2-p53 feedback loop, resulting in sensitivity of cancer cells to anticancer treatment. Furthermore, suppression of XIAP following FKBP12-mediated MDM2 degradation represents an additional mechanism contributing to enhanced sensitivity. FKBP12-mediated MDM2 degradation and subsequent suppression of XIAP are p53-independent. Therefore, this mechanism may play roles in cancers with different p53 background. This is significantly important. It has been shown that most cancer patients, including breast cancer patients, do not carry wild-type p53 [26]. In addition, the incidence of $\mathrm{p} 53$ loss or mutation is obviously higher in aggressive cancer [27-29].

Although FKBP12 is widely expressed in normal cells/ tissues, its expression in cancer cells/tissues has not been extensively studied. A recent study found that the expression level of FKBP12 varied significantly among different cancer cell lines [30]. We further observed a negative 
correlation between FKBP12 and MDM2 expression, which determined the responsiveness of cancer cells to anticancer treatment [12]. Here, we validated these observations in human breast cancer and indicated that FKBP12-MDM2 is predictive for therapeutic outcome. We provide a systematic investigation of the clinical relevance of FKBP12.

In summary, we propose that FKBP12 loss, which can be enhanced by MDM2 overexpression, predicts poor prognosis and chemoresistance. By future large prospective, randomized, controlled studies, the therapeutic strategy increasing the expression of FKBP12 may be a valuable addition to chemotherapy, especially in MDM2overexpressed patients.

Acknowledgements We thank Prof. Renliang Wu for revising the manuscript. We would like to thank Editage (www.editage.cn) for English language editing.

Authors'contributions MY-X, JX, JS-L, and SZ designed and performed experiments, and analyzed the data. JX and JW provided pathology guidelines and samples. QY helped with experimental design and analysis of data. YW and SZ designed experiments, analyzed data, supervised the study, and wrote the manuscript. All authors read and approved the final manuscript.

Funding This study was supported by the National Nature Science Foundation of China (Grant nos. 81874117, 81472488, and 81502296).

Data availability The data sets used and/or analyzed during the current study are available from the corresponding author on reasonable request.

\section{Compliance with ethical standards}

Conflict of interest All authors declare that he/she has no conflict of interest.

Ethical approval All procedures performed in studies involving human participants were in accordance with the ethical standards of the institutional and/or national research committee and with the 1964 Helsinki Declaration and its later amendments or comparable ethical standards. This research was approved by the Ethics Committee of Tongji Hospital, Huazhong University of Science and Technology.

Informed consent Informed consent was obtained from all individual participants included in this study.

Open Access This article is distributed under the terms of the Creative Commons Attribution 4.0 International License (http://creativeco mmons.org/licenses/by/4.0/), which permits unrestricted use, distribution, and reproduction in any medium, provided you give appropriate credit to the original author(s) and the source, provide a link to the Creative Commons license, and indicate if changes were made.

\section{References}

1. Bianchini G, Balko JM, Mayer IA, Sanders ME, Gianni L (2016) Triple-negative breast cancer: challenges and opportunities of a heterogeneous disease. Nat Rev Clin Oncol 13(11):674-690

2. Foulkes WD, Smith IE, Reis-Filho JS (2010) Triple-negative breast cancer. N Engl J Med 363(20):1938-1948

3. Mayer EL, Burstein HJ (2016) Chemotherapy for triple-negative breast cancer: is more better? J Clin Oncol 34(28):3369-3371

4. Harding MW, Galat A, Uehling DE, Schreiber SL (1989) A receptor for the immunosuppressant FK506 is a cis-trans peptidyl-prolyl isomerase. Nature 341(6244):758-760

5. Bierer BE, Mattila PS, Standaert RF, Herzenberg LA, Burakoff SJ, Crabtree G, Schreiber SL (1990) Two distinct signal transmission pathways in $\mathrm{T}$ lymphocytes are inhibited by complexes formed between an immunophilin and either FK506 or rapamycin. Proc Natl Acad Sci USA 87(23):9231-9235

6. Liu J, Farmer JD Jr, Lane WS, Friedman J, Weissman I, Schreiber SL (1991) Calcineurin is a common target of cyclophilin-cyclosporin A and FKBP-FK506 complexes. Cell 66(4):807-815

7. Sabers CJ, Martin MM, Brunn GJ, Williams JM, Dumont FJ, Wiederrecht G, Abraham RT (1995) Isolation of a protein target of the FKBP12-rapamycin complex in mammalian cells. J Biol Chem 270(2):815-822

8. Lorenz MC, Heitman J (1995) TOR mutations confer rapamycin resistance by preventing interaction with FKBP12-rapamycin. J Biol Chem 270(46):27531-27537

9. Brillantes AB, Ondrias K, Scott A, Kobrinsky E, Ondriasová E, Moschella MC, Jayaraman T, Landers M, Ehrlich BE, Marks AR (1994) Stabilization of calcium release channel (ryanodine receptor) function by FK506-binding protein. Cell 77(4):513-523

10. Wang T, Li BY, Danielson PD, Shah PC, Rockwell S, Lechleider RJ, Martin J, Manganaro T, Donahoe PK (1996) The immunophilin FKBP12 functions as a common inhibitor of the TGF beta family type I receptors. Cell 86(3):435-444

11. Mathea S, Li S, Schierhorn A, Jahreis G, Schiene-Fischer C (2011) Suppression of EGFR autophosphorylation by FKBP12. Biochemistry 50(50):10844-10850

12. Liu T, Xiong J, Yi S, Zhang H, Zhou S, Gu L, Zhou M (2017) FKBP12 enhances sensitivity to chemotherapy-induced cancer cell apoptosis by inhibiting MDM2. Oncogene 36(12):1678-1686

13. Turbin DA, Cheang MC, Bajdik CD, Gelmon KA, Yorida E, De Luca A, Nielsen TO, Huntsman DG, Gilks CB (2006) MDM2 protein expression is a negative prognostic marker in breast carcinoma. Mod Pathol 19(1):69-74

14. Rayburn E, Zhang R, He J, Wang H (2005) MDM2 and human malignancies: expression, clinical pathology, prognostic markers, and implications for chemotherapy. Curr Cancer Drug Targets 5(1):27-41

15. Momand J, Zambetti GP, Olson DC, George D, Levine AJ (1992) The mdm-2 oncogene product forms a complex with the $\mathrm{p} 53$ protein and inhibits p53-mediated transactivation. Cell 69(7):1237-1245

16. Honda R, Tanaka H, Yasuda H (1997) Oncoprotein MDM2 is a ubiquitin ligase E3 for tumor suppressor p53. FEBS Lett 420:25-27

17. Juven T, Barak Y, Zauberman A, George DL, Oren M (1993) Wild type p53 can mediate sequence-specific transactivation of an internal promoter within the mdm2 gene. Oncogene 8(12):3411-3416

18. Zhang X, Gu L, Li J, Shah N, He J, Yang L, Hu Q, Zhou M (2010) Degradation of MDM2 by the interaction between berberine and DAXX leads to potent apoptosis in MDM2-overexpressing cancer cells. Cancer Res 70(23):9895-9904 
19. Zhang Z, Zhang R (2005) p53-independent activities of MDM2 and their relevance to cancer therapy. Curr Cancer Drug Targets 5(1):9-20

20. Gu L, Zhu N, Zhang H, Durden DL, Feng Y, Zhou M (2009) Regulation of XIAP translation and induction by MDM2 following irradiation. Cancer Cell 15(5):363-375

21. Gu L, Zhang H, Liu T, Zhou S, Du Y, Xiong J, Yi S, Qu CK, Fu H, Zhou M (2016) Discovery of dual inhibitors of MDM2 and XIAP for cancer treatment. Cancer Cell 30(4):623-636

22. Zhou S, Gu L, He J, Zhang H, Zhou M (2011) MDM2 regulates vascular endothelial growth factor mRNA stabilization in hypoxia. Mol Cell Biol 31(24):4928-4937

23. Xiong J, Yang Q, Li J, Zhou S (2014) Effects of MDM2 inhibitors on vascular endothelial growth factor-mediated tumor angiogenesis in human breast cancer. Angiogenesis 17(1):37-50

24. Singh S, Ramamoorthy M, Vaughan C, Yeudall WA, Deb S, Palit Deb S (2013) Human oncoprotein MDM2 activates the Akt signaling pathway through an interaction with the repressor element-1 silencing transcription factor conferring a survival advantage to cancer cells. Cell Death Differ 20(4):558-566

25. Xiong J, Su T, Qu Z, Yang Q, Wang Y, Li J, Zhou S (2016) Triptolide has anticancer and chemosensitization effects by downregulating Akt activation through the MDM2/REST pathway in human breast cancer. Oncotarget 7(17):23933-23946
26. Hollstein M, Sidransky D, Vogelstein B, Harris CC (1991) p53 mutations in human cancers. Science 253(5015):49-53

27. Mazars R, Spinardi L, BenCheikh M, Simony-Lafontaine J, Jeanteur P, Theillet C (1992) p53 mutations occur in aggressive breast cancer. Cancer Res 52(14):3918-3923

28. Bourdon JC, Khoury MP, Diot A, Baker L, Fernandes K, Aoubala M, Quinlan P, Purdie CA, Jordan LB, Prats AC, Lane DP, Thomp-

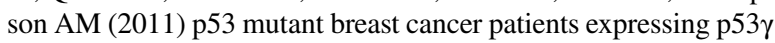
have as good a prognosis as wild-type p53 breast cancer patients. Breast Cancer Res 13(1):R7

29. Lu X, Liu DP, Xu Y (2013) The gain of function of p53 cancer mutant in promoting mammary tumorigenesis. Oncogene 32(23):2900-2906

30. Schreiber KH, Ortiz D, Academia EC, Anies AC, Liao CY, Kennedy BK (2015) Rapamycin-mediated mTORC2 inhibition is determined by the relative expression of FK506-binding proteins. Aging Cell 14(2):265-273

Publisher's Note Springer Nature remains neutral with regard to jurisdictional claims in published maps and institutional affiliations. 